\title{
Effects of a task-irrelevant stimulus dimension on asymptotic response probability in children
}

\author{
SILKE VOGELMANN and LEONARD P. ULLMANN \\ University of Hawaii, Honolulu, Hawaii 96822
}

\begin{abstract}
The effects of the distribution (3:1 or $1: 3$ ) of the task-irrelevant stimulus dimension (color or form) on asymptotic choice proportions of the $75 \%$ task-relevant stimulus in a noncontingent two-choice probability learning situation were investigated. Children of two age groups (3 and 11 years old) served as subjects. The results in predicting the more frequent alternative $(75 \%)$ indicated that: (1) The 3-year-old children showed little evidence of change across trials and did not reach the matching value; (2) the 11-year-old children showed increasing choice proportions until stabilizing at the matching level; (3) reinforcement was not effective in raising response proportions for either age group; (4) both ages showed higher response proportions when the color rather than the form stimulus was task relevant; and (5) no evidence was obtained for differential effects of the dimension or the probability distribution of the task-irrelevant stimulus.
\end{abstract}

The present study was designed to investigate the effects of a task-irrelevant (T-I) stimulus dimension (color or form) on the asymptotic response probability in children in a noncontingent two-choice situation (Estes \& Straughan, 1954; Humphreys, 1939). In particular the study was concerned with the following question: Do variations in the distributions of a T-I stimulus dimension $(3: 1$ vs $1: 3)$ influence the probability of predicting the occurrence of the more frequent task-relevant (T-R) stimulus dimension which consistently occurs with a 75:25 distribution? The T-I stimulus is defined as a variable which is irrelevant to the subject's encoding and response execution components of information processing. The experimental task is the probability learning situation of McInnis and Ullmann (1967) and Ullmann and Straughan (Note 1) with two modifications: (1) Children are used as subjects and (2) T-I and T-R stimulus dimensions are presented simultaneously. For example, if form is T-R with $75 \%$ triangles and $25 \%$ squares presented, color is T-I. Thus, in the $3: 1$ situation the $75 \%$ alternative is subdivided into equal proportions of red, green, and blue triangles. The squares occur in only one type of color. However, in the $1: 3$ situation the $75 \%$ alternative occurs in one color and the squares are presented in equal proportions of red, green, and blue. The subject predicts whether a circle or square will occur and observes after each trial the correctness of his response. If color is the $\mathrm{T}-\mathrm{R}$ dimension, form is T-I and occurs with a $1: 3$ or $3: 1$ distribution.

The present design addresses two issues: (1) dimensional preference in children of different ages and (2) probability learning. The first investigates the effects

The authors are grateful to the principals and teachers of the Kokohead Elementary School and of the Pali and Manoa Valley Preschools for their cooperation in this study. of possible differences in attentional processes in children of different ages. It has been suggested that European and American children show a shift from color to form preference on forced-choice tasks between ages 4 and 5 years (Brian \& Goodenough, 1929; Cole, Gay, Glick, \& Sharp, 1971; Cole \& Scribner, 1974; Descoudres, 1914; Serpell, 1969; Suchman, 1966; Suchman \& Trabasso, 1966). Within the present context it is hypothesized that the salience of the T-I stimulus dimension should be age related. Children under 4 years should be more sensitive to color and older children to form. Specific effects of the T-I stimulus dimension would be expected to vary with age. More precise hypotheses are discussed below. The effects of reinforcing children for correct predictions are also investigated. Previous research on response asymptotes of children in the noncontingent two-choice situation has shown that under conditions of reinforcement (R) and nonreinforcement (NR) younger children (ages 3.0 to 6.5 years) tended to show a lower choice asymptote than older children (ages 9.0 to 17.0 years) (Craig \& Myers, 1963; Crandall, Solomon, \& Kellaway, 1961; Messick \& Solley, 1957; Offenbach, 1964). Only Jones and Liverant (1960) found evidence that younger children (4.0 to 6.0 years old) tended to manifest a higher asymptotic response probability than older children (9.0 to 11.0 years old) under $R$. If $R$ only consists of observing the correctness of one's predictions (so-called NR condition), evidence exists that children ranging from 3 to 12 years tend to match their response asymptotes to the actual probability of occurrence of the events or fall below the matching value (Brackbill, Kappy, \& Starr, 1962, Craig \& Myers, 1963; Crandall et al., 1961; Messick \& Solley, 1957; Offenbach, 1964; Siegel \& Andrews, 1962). If $\mathrm{R}$ is introduced to children ranging in ages from 3 to 17 years, some studies indicate that response asymptotes exceed the matching value 
(Brackbill et al., 1962; Jones \& Liverant, 1960; Messick \& Solley, 1957; Siegel \& Andrews, 1962), whereas other studies indicate that response asymptotes remain at or below the matching level (Crandall et al., 1961; Jones \& Liverant, 1960; Messick \& Solley, 1957; Offenbach, 1964). It is difficult to evaluate the discrepancy of these findings since studies tend to vary with respect to variables such as operational definition of response asymptote, task materials utilized, types and amounts of $\mathbf{R}$, and age groups studied.

Given the previous research findings, the following hypotheses were formulated with respect to the asymptotic response probability, defined as the number of choices of the more frequent T-R stimulus during the second 100 trials (101-200) of the probability learning situation as previously used by McInnis and Ullmann (1967) and Ullmann and Straughan (Note 1). (1) Older children (11 years) show a higher response asymptote than younger children (3 years). (2) For all conditions, the presence of $R$ induces a higher response asymptote than the absence of $R$. (3) It is predicted that the two probability distributions $(1: 3$ vs $3: 1)$ of the T-I stimulus exert differential effects on the response asymptotes. Therefore, a significant T-I stimulus main effect is predicted. (4) Any effect of the probability distribution of the T-I stimulus dimension should vary with the age and type of dimension (color vs form) used. A significant T-R Stimulus by T-I Stimulus by Age interaction is predicted.

\section{METHOD}

\section{Subjects}

The sample consisted of 64 children of two different age groups. Thirty-two children (17 girls, 15 boys) had a mean age of 141.5 months with a SD of 4.4 months, and the remaining 32 children (17 girls, 15 boys) had a mean age of 42.6 months with a SD of 3.6 months. The older children were obtained from the sixth grade of an elementary school and the younger children from two preschools in Honolulu. The children were of varied ethnic background (predominantly Oriental). In order to be included in the analysis, subjects had to attend to the task, follow instructions, and complete the task within $1.5 \mathrm{~h}$. Twelve of the younger children did not meet this criterion and were replaced.

\section{Materials}

White unlined $3 \times 5$ in. cards of double thickness were used to assure complete opaqueness. Triangles, circles, or squares of different colors (red, green, or blue) were placed in the center of each card. The stimuli (pressure-sensitive geo shapes No. 98.8622) were manufactured by the Mead Corporation, Atlanta. The diameter of the circle was $2.02 \mathrm{~cm}$, the altitude of the equilateral triangle was $2.02 \mathrm{~cm}$, and a side of the square was $2 \mathrm{~cm}$. A 200 -card deck consisting of 10 blocks of 20 cards was prepared for each subject and consisted of the specific stimulus combination as specified in the Design section. The ratio of the T-R stimuli was fixed within each block of 20 trials at $75: 25$ and of the T-I stimuli at $1: 3$ or $3: 1$ (see Design section). Different randomized series of events were constructed in blocks of 20 trials each. A small practice deck consisting of 10 cards was prepared for each subject. In addition to the pack of cards, a box containing approximately 200 beads (back-up reinforcers) and a cup with a 200-bead capacity, in which the subject ac- cumulated his "winnings," were placed on the table at which the subject and the experimenter worked. On display in another part of the room was a box containing various candies and approximately 80 small toys, such as cars, necklaces, balloons, and rings. The accumulated beads could be exchanged for a toy or candy.

\section{Design}

The study was a 2 by 2 by 2 by 2 factorial design with age (3 or 11 years), R (presence or absence), T-R stimulus (color or form), and T-I stimulus distribution (proportion of $3: 1$ or $1: 3)$ as the four between-subjects factors. The ratio of types of the T-R stimulus dimension was always fixed at $75: 25$ within a block of 20 trials. The order of stimulus presentation was randomized. If form represented the T-R dimension, color was $T-I$ and vice versa. For each T-R dimension the type of form (triangle or square) or color (red or blue) used was counterbalanced within each experimental group. Likewise, the position of the sample card indicating the $75 \%$ alternative to which the child had to point in order to make his choice (right or left) was counterbalanced within each experimental group. In the $3: 1$ condition the $75 \%$ alternative was subdivided into equal proportions of three different colors or forms, whereas the $25 \%$ alternative consisted of only one type of color or form. In the $1: 3$ condition the distributions were reversed, with the $25 \%$ alternative being divided into equal proportions of three different colors (red, blue, green) or forms (triangle, square, circle) and the $75 \%$ alternative appearing in only one type of color or form. The type of stimulus used for the undivided alternative was counterbalanced within each experimental group. As only four subjects per group were used for the present study, each subject received a unique stimulus combination (as a result of counterbalancing various variables).

\section{Procedure}

Subjects within each age group were randomly assigned to the experimental conditions. There was a total of 16 groups with four subjects per group. Sixteen experimenters were randomly assigned to the subjects (four children per experimenter). Each experimenter was to test additional subjects if one of his/her subjects could not be used for the analysis. All subjects were tested individually. The pack of 200 cards and the practice deck were placed face down on the table. The child also received two sample cards on which only the types of the T-R stimulus dimension were represented. If the T-R dimension was form, two cards, one with a yellow square and the other with a yellow triangle, were presented. If the dimension was color, a red and a blue cross were presented as sample cards. Sample cards were used in order that subjects could make their guesses nonverbally by simply pointing to one of the two cards. After the subject made his/her choice, the top card of the 200-card deck was turned over for his/her inspection and then placed at the bottom of the pack. In this way the child was unable to predict the end of the session. The child was told that the task was a guessing game. In the $\mathrm{R}$ condition the child was further told that he/she would obtain one of the beads each time he/she made a correct prediction. If he/she had "won" enough beads at the end of the session, he/she could exchange them for a candy or toy. Thus, it was important to get as many correct as possible.

Children in the NR condition received a noncontingent reward, for example, they could choose a toy or candy before the start of the session. They were told that they could select a candy or toy because they were participating in the game. This procedure was used to insure that all children felt they were treated in an equal manner. After the instructions were given, the experimenter administered practice trials and repeated instructions until it was evident that the child understood the task. Great care was taken not to induce a set in the children by labeling the relevant stimulus dimension or its associated types, for example, color, form, red, blue, triangle, or square. If a child, after comparing the sample stimuli with the experimental 
stimuli, remarked that there were no yellow squares or red crosses, an ambiguous answer was given, namely, that this was true, but that these two (the sample and experimental cards) were alike in some other way.

Children in the NR condition were presented with noncontingent verbal and nonverbal positive R: "That is fine," "Very good," "You're doing well," encouraging smiles, and so forth. In order to insure that these rewards were given noncontingently, they were presented prior to the start of the next trial but quite some time after the end of the previous trial. Children in the $\mathbf{R}$ condition recieved verbal $R$ s contingent on correct guesses only. In addition, they obtained the beads. Noncontingent rewards were used to insure adequate motivation throughout. Rest periods were permitted whenever subjects appeared to be tiring. However, the task had to be completed within $1.5 \mathrm{~h}$.

\section{RESULTS}

A repeated measures analysis of variance with 10 successive 20-trial blocks as the repeated factor was conducted. The results indicated that the Age by Blocks of Trials interaction was significant $[F(9,432)=4.029$, $\mathrm{p}<.001]$. The means are presented in Table 1. Differences between the groups could be accounted for by a significant linear $[F(1,48)=10.816, p<.01]$ and quadratic trend $[\mathrm{F}(1,48)=6.293, \mathrm{p}<.02]$. The linear component accounted for $61 \%$ of the variance and the quadratic for $21 \%$ of the variance. This suggests that most of the variance results from the 3-year-old children showing virtually no improvement in predicting the occurrence of the $75 \%$ alternative across blocks of trials, whereas the 11-year-old children showed an increasing function which reached asymptote after approximately 140 trials. An analysis of variance with the mean number of responses across the first and second hundred trials (Trials 1-100 and 101-200) as the repeated factor showed that the Age by Blocks of Trials interaction was significant $[F(1,48)=15.754, p<.0003]$. NewmanKeuls tests confirmed that the difference between the means of the first (.62) and second (.64) hundred trials was not significant for the 3-year-olds. However, the difference between the means $(.62$ and .75$)$ for the 11 -year-olds was highly significant $(p<.01)$. This result substantiates differences in performance across blocks of trials between the two age groups. A further analysis of variance of the proportion of choices of the more frequent alternative during the second hundred trials (asymptotic response proportion) indicated that the age main effect was significant $[\mathrm{F}(1,48)=11.91$, $\mathrm{p}<.002]$. The means revealed that the 11 -year-old

Table 1

Mean Proportions of Predicting the 75\% Task-Relevant Stimulus in Successive 20-Trial Blocks for the Two Age Groups

\begin{tabular}{ccccccccccc}
\hline & \multicolumn{10}{c}{ Blocks } \\
\cline { 2 - 11 } Age & 1 & 2 & 3 & 4 & 5 & 6 & 7 & 8 & 9 & 10 \\
\hline 3 & .58 & .58 & .63 & .61 & .65 & .61 & .62 & .66 & .65 & .68 \\
11 & .52 & .60 & .65 & .66 & .70 & .72 & .76 & .73 & .74 & .77 \\
\hline
\end{tabular}

Note-Age is in years. children showed matching (.75), while the 3-year-olds underestimated the occurrence of the more frequent alternative (.64). The effect of the T-R stimulus dimension was also significant $[F(1,48)=5.61, p<.03]$. The means indicated that the asymptotic response probability was significantly lower when the T-R stimulus was form (.66) than when it was color (.73). It was also found that 10 children showed a response asymptote higher than .80 under the color condition; only two children performed in the same fashion in the form condition.

\section{DISCUSSION}

The study found positive evidence for differences with respect to asymptotic choice proportion and rate of acquisition in 3- and 11-year-old children in a noncontingent two-choice probability learning situation. During the learning phase the younger children showed little evidence of change in predicting the occurrence of the more frequent alternative. They predicted the $75 \%$ alternative with a mean probability which was above .50 (chance level) but below the matching value. The 11-year-old children demonstrated increasing choice proportions until their mean performance stabilized around the matching value. Thus, positive evidence was obtained for Hypothesis 1 . These results are also in agreement with Craig and Myers (1963) and Crandall et al. (1961). Informal observations suggested that 3-year-olds process information on a more primitive level. They seemed to notice that two types of stimuli occurred and that they varied. But they were less sensitive to the differential frequencies of occurrence of these two events than were the older children. The presence of reinforcement was not effective in raising response asymptotes. These results may be interpreted as providing support for Estes' (1964) view regarding the noncontingent situation. He maintains that a major factor for response asymptotes to exceed the matching value is not reinforcement per se, but rather the instructions focusing the subject's attention on certain aspects of the situation. No evidence was obtained in support of (1) a differential effect of the probability distribution of the T-I stimulus dimension and (2) the predicted Age by T-R Stimulus by T-I Stimulus interaction. Apparently, the effect of an independent probability distribution of a second stimulus which is not relevant per se for response execution had no influence. The present results furthermore imply that no evidence was obtained for differences in response asymptotes due to dimensional preferences for color or form which are expected to be different for the two age groups.

A rather unexpected finding was that children of both age groups tended to respond, on the average, with higher response asymptotes when the T-R stimulus was color rather than form. Further research is needed to investigate (1) whether this phenonmenon is reliable and (2) some of the causally associated factors. From a theoretical perspective, the present results are interesting in light of Estes' (1976) work. Frequencies of occurrence of stimuli presented were controlled for the T-R stimulus dimension (color or form) and thus could not account for the present results. In this context, beyond the results themselves, the present work indicates how the probability learning situation may permit the evaluation of the salience of different stimulus dimensions on different populations.

\section{REFERENCE NOTE}

1. Ullmann, L. P., \& Straughan, J. S. Probability learning in schizophrenic males. Paper read at Western Psychological Association, San Diego, April 1959. 


\section{REFERENCES}

Brackbill, Y., Kappy, M. S., \& StarR, R. H. Magnitude of reward and probability learning. Journal of Experimental Psychology, 1962, 63, 32-35.

Brian, C., \& Goodenough, F. Relative potency of color and form perception at various ages. Journal of Experimental Psychology, 1929, 12, 197-213.

Cole, M., GAY, J., Glick, J., \& Sharp, D. The cultural context of learning and thinking. New York: Basic Books, 1971.

Cole, M., \& SCribner, S. Culture and thought. New York: John Wiley, 1974.

Craig. G. J.. \& Myers, J. L. A developmental study of sequential two-choice decision making. Child Development, 1963, 34, 483-493.

Crandall, V. J., Solomon, D., \& Kellaway, R. A comparison of the patterned and nonpatterned probability learning of adolescent and early school-age children. Journal of Genetic Psychology, 1961, 99, 29-39.

Descoudres, A. Couleur, forme, ou nombre? Archives of Psychology, 1914, 14, 305-341.

Estes, W. K. Probability learning. In A. W. Melton (Ed.), Categories of human learning. New York: Academic Press, 1964.

Estes, W. K. The cognitive side of probability learning. Psychological Review, 1976, 83, 37-64.

Estes, W. K., \& Straughan, J. H. Analysis of a verbal conditioning situation in terms of statistical learning theory. Journal of Experimental Psychology, 1954, 47. 225-234.
Humphreys, L. G. Acquisition and extinction of verbal expectations in a situation analogous to conditioning. Journal of Experimental Psychology, 1939, 25, 294-301.

Jones, M. H., \& Liverant, S. Effects of age differences on choice behavior. Child Development, 1960, 31, 673-680.

McInnis, T. L., \& UllmanN, L. P. Positive and negative reinforcement with short- and long-term hospitalized schizophrenics in a probability learning situation. Journal of Abnormal Psychology, 1967, 2, 157-162.

Messick, S. J., \& Solley, M. C. Probability learning in children: Some exploratory studies. Journal of Genetic Psychology, 1957, 90, 23-32.

OfFEnBach, S. I. Studies of children's probability learning behavior: I. Effect of reward and punishment at two age levels. Child Development, 1964, 35, 709-715.

Serpell. R. Cultural differences in attentional preference for color over form. International Journal of Psychology, 1969, 4, $1-8$

Siegel. S., \& Andrews, J. M. Magnitude of reinforcement and choice behavior in children. Journal of Experimental Psychology, 1962, 63, 337-341.

Suchman, R. Cultural differences in children's color and form preferences. Journal of Social Psychology, 1966 , 70, 3-10.

Suchman, R., \& Trabasso, 'T. Color and form preference in young children. Journal of Experimental Psychology, 1966. 3, 177-187. 